\title{
Integral equation methods with unique solution for all wavenumbers applied to acoustic radiation
}

\author{
Antoine Lavie - Alexandre Leblanc \\ Univ Lille Nord de France - UArtois \\ Laboratoire Génie Civil et géo-Environnement (LGCgE) \\ Technoparc Futura \\ F-62400 Béthune \\ \{antoine.lavie,alexandre.leblanc\}@univ-artois.fr
}

ABSTRACT. The acoustic exterior Neumann problem is solved using an easy process based upon the boundary element method and able to eliminate effects of irregular frequencies in time harmonic domain. This technique is performed as follows: (i) two computations are done around the characteristic frequency, decreased and increased by a small imaginary part; (ii) average between pressures at these two frequencies ensures unique solution for all wavenumbers. This method is numerically tested for an infinite cylinder, an axisymmetric cylinder, a sphere and a three-dimensional cat's eye structure. This work highlights ease and efficiency of the technique under consideration to remove the irregular frequencies effects.

RÉSUMÉ. Le problème acoustique de Neumann extérieur est résolu par une technique numérique facile à mettre en œuvre basée sur une méthode d'éléments de frontière dans le régime harmonique. Cette méthode est capable d'éliminer les effets des fréquences irrégulières. La technique se décompose en deux étapes : (i) deux calculs sont effectués autour de la fréquence à traiter (augmentée ou diminuée par une petite partie imaginaire) ; (ii) moyennage de la pression entre ces deux fréquences. Cette démarche permet d'assurer l'unicité de la solution à la fréquence traitée. La méthode est testée sur un cylindre infini, un cylindre à symétrie axiale, une sphère et une géométrie tridimensionnelle type " ail de chat». Ce travail démontre la simplicité et l'efficacité de la technique proposée pour traiter l'indétermination aux fréquences irrégulières.

KEYWORDS: acoustic radiation, boundary element method, irregular frequencies.

MOTS-CLÉS: rayonnement acoustique, méthode des éléments de frontière, fréquences irrégulières.

DOI:10.3166/EJCM.19.619-636 @ 2010 Lavoisier, Paris

EJCM - 19/2010. Fluid-structure interaction, pages 619 to 636 


\section{Introduction}

The boundary element method is well-suited to the study of the acoustic radiation or scattering from any arbitrary body immersed in a fluid medium of infinite extension (Marburg et al., 2008). Here, the 3D Helmholtz equation is solved with a prescribed Neumann boundary condition on the radiated or scattered surface, through the so-called exterior Helmholtz integral equation (Schenck, 1968) (HIE) and the wave superposition method (Koopmann et al., 1989) (WSM). In our case, the nonuniqueness difficulty occurs at the eigenfrequencies of the associate interior Dirichlet problem (Schenck, 1968, Copley, 1968). Numerically, this means that at these eigenfrequencies and in their neighborhood, the final set of equations is singular. The lack of uniqueness of solution at these frequencies can be overcome by alternative techniques, such those initially suggested by Schenck (1968), Burton et al. (1971), Waterman (1969) or Jones (1974) (Benthien et al., 1997, have compared efficiency of these methods to treat irregular frequencies effects). The first two methods have benefited from numerous studies. The first one is very popular and is known by the acronym CHIEF (Combined Helmholtz Integral Equation Formulation). This method combines the HIE with collocation points in the interior domain of the scattering body. By adding the HIE of the interior domain, CHIEF creates an overdetermined system of equations, which can be solved using a least-square technique. It has been improved by many authors (Seybert et al., 1987, Segalman et al., 1990, Wu et al., 1991, Segalman et al., 1992, Marschall, 1993, Juhl, 1994, Chen et al., 1997). The second method uses a complex linear combination of the HIE and its normal derivative equation (which leads to hypersingular kernel). This technique yields an unique solution when the coupling coefficient is a complex number. The main difficulties have been resolved for many years (Meyer et al., 1978, Yang, 2004). The Burton and Miller method is also attractive and can largely compete with Schenck's method (Marburg et al., 2005). In the numerical application of these methods there is always a tradeoff between accuracy and speed, as there is often a tradeoff between complexity (difficulty of implementation) and reliability (protection against failure) (Benthien et al., 1997). Thus, we propose a new process which is accurate, easy to implement and reliable.

The first part of this paper deals with the acoustic problem: the exterior Neumann problem and the solutions we have developed are summarized and irregular frequencies are briefly remembered. Then, the proposed process to overcome irregular frequencies is described. The second part concerns numerical investigations of the new method which are based on the point-source check. Infinite cylinder and axisymmetric geometries (cylinder and sphere) and their discretization are defined, computations are carried out with WSM and classical HIE and with special treatment. In order to apply this technique to more 3D complex problem, the cat's eye (Marburg et al., 2003, Schneider et al., 2003, Marburg et al., 2005, Marburg et al., 2008, Makarov et al., 1998) is tested. Finally, conclusion is done. 


\section{Acoustic problem}

\subsection{Equations of the problem}

Figure 1 shows the geometry of the radiation problem. The well-known Helmholtz differential equation governs the propagation in infinite fluid domain:

$$
\Delta p(\boldsymbol{r})+k^{2} p(\boldsymbol{r})=0 \quad \boldsymbol{r} \in \Omega_{f},
$$

where $p$ is the amplitude of the acoustic pressure at the point $r$ and $k$ is the wavenumber. The variables exhibit an implicit $e^{-i \omega t}$ dependence where $\omega=k c$ ( $c$ is the sound velocity) is the pulsation and $t$ the time $(i=\sqrt{-1})$. This equation is associated with the Neumann boundary condition on the surface:

$$
\frac{\partial p\left(\boldsymbol{r}^{\prime}\right)}{\partial \boldsymbol{n}^{\prime}}=i \omega \rho v_{n}\left(\boldsymbol{r}^{\prime}\right) \quad \boldsymbol{r}^{\prime} \in \Gamma
$$

in which $\partial \boldsymbol{n}^{\prime}$ denotes normal differentiation at the point $\boldsymbol{r}^{\prime}$ in the direction from the interior region $\Omega_{i}$ towards $\Gamma, v_{n}\left(\boldsymbol{r}^{\prime}\right)$ is the normal component of the velocity at point $\boldsymbol{r}^{\prime}$ and $\rho$ is the fluid density modulus. The pressure must also satisfy the radiation condition (Wilcox, 1956):

$$
\lim _{R \rightarrow \infty} \iint_{S_{R}}\left|\frac{\partial p(\boldsymbol{r})}{\partial r}-i k p(\boldsymbol{r})\right|_{r=R}^{2} d S=0,
$$

where $r$ is the radial distance from the origin of coordinates and $S_{R}$ is a sphere of radius $R$ centered at the origin and surrounding $\boldsymbol{r}$ and $\Gamma$.

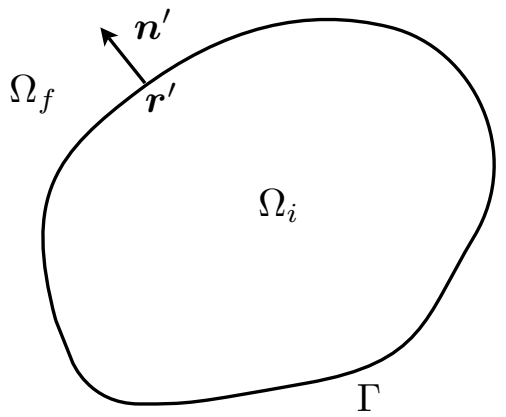

Figure 1. Geometry of the problem

\subsubsection{Helmholtz integral equation}

The above problem can be replaced by the HIE (Schenck, 1968, Brebbia, 1991):

$$
\frac{\alpha(\boldsymbol{r})}{4 \pi} p(\boldsymbol{r})=\iint_{\Gamma}\left(p\left(\boldsymbol{r}^{\prime}\right) \frac{\partial g\left(\boldsymbol{r}, \boldsymbol{r}^{\prime}\right)}{\partial \boldsymbol{n}^{\prime}}-\frac{\partial p\left(\boldsymbol{r}^{\prime}\right)}{\partial \boldsymbol{n}^{\prime}} g\left(\boldsymbol{r}, \boldsymbol{r}^{\prime}\right)\right) \boldsymbol{d} \boldsymbol{r}^{\prime},
$$


where $\alpha$ is the solid angle (Seybert et al., 1985) (equal to $2 \pi$ when $r$ is a regular point on $\Gamma$, equal to 0 when $r \in \Omega_{i}$ and equal to $4 \pi$ when $r \in \Omega_{f}$ ) and $g$ is the free-space Green's function:

$$
\begin{aligned}
& g\left(\boldsymbol{r}, \boldsymbol{r}^{\prime}\right)=H_{0}^{(1)}\left(k\left|\boldsymbol{r}-\boldsymbol{r}^{\prime}\right|\right) \\
& g\left(\boldsymbol{r}, \boldsymbol{r}^{\prime}\right)=\frac{1}{4 \pi} \frac{e^{i k\left|\boldsymbol{r}-\boldsymbol{r}^{\prime}\right|}}{\left|\boldsymbol{r}-\boldsymbol{r}^{\prime}\right|}
\end{aligned}
$$

in which $H_{0}^{(1)}$ is the Hankel function of the first kind, $\left|\boldsymbol{r}-\boldsymbol{r}^{\prime}\right|$ is the distance between points $r$ and $\boldsymbol{r}^{\prime}$. The discretization of the surface using isoparametric elements with quadratic variation leads to the linear square system (cf. Stupfel and al. (Stupfel et al., 1988) for the axisymmetric case and Lavie (Lavie, 1989) for the 3D case):

$$
[A]\{p\}=[B]\left\{\frac{\partial p}{\partial n}\right\}
$$

where $[A]$ and $[B]$ are the integral equation matrices, $\{p\}$ and $\{\partial p / \partial n\}$ are the nodal pressures and nodal derivative pressures vectors. After calculation of the surface pressure, near-field pressure and far-field pressure can be directly deduced.

\subsubsection{The wave superposition method}

The simple idea behind the WSM is that the acoustic field of a complex radiator can be reconstructed as a superposition of individual simple sources. Its principle can be found in the literature under various denominations as the well known Method of Fundamental Solutions (MFS) (Fairweather et al., 2003). The equivalency of the WSM to the HIE has been shown by Koopman (Koopmann et al., 1989), thus validating the superposition integral:

$$
p(\boldsymbol{r})=j \omega \rho \iint_{\Omega_{i}} q\left(\boldsymbol{r}_{w}\right) g\left(\boldsymbol{r}, \boldsymbol{r}_{w}\right) \boldsymbol{d} \boldsymbol{\Omega}_{\boldsymbol{i}}\left(\boldsymbol{r}_{\boldsymbol{w}}\right) \quad \boldsymbol{r} \in \Gamma \cup \Omega_{f} .
$$

The source strength is denoted by $q\left(\boldsymbol{r}_{w}\right)$ where $\boldsymbol{r}_{w}$ are the WSM source locations inside $\Omega_{i}$.

To reduce Equation [8] to a numerical form, it's convenient to assume the sources distributed on $\Gamma^{\prime}$ inside $\Omega_{i}$. If this surface is divided into $N$ sufficiently small elements, the normal velocity on $\Gamma$ can be approximated:

$$
u_{n}(\boldsymbol{r}) \approx \sum_{i=1}^{N} Q_{i} \frac{\partial g\left(\boldsymbol{r}, \boldsymbol{r}_{w_{i}}\right)}{\partial \boldsymbol{n}} \quad \boldsymbol{r} \in \Gamma \cup \Omega_{f} \quad \boldsymbol{r}_{w_{i}} \in \Gamma^{\prime},
$$

where $Q_{i}$ is the volume velocity of the simple source. Since $u_{n}(\boldsymbol{r})$ is known on $\Gamma, Q_{i}$ is given in matrix form by

$$
Q=[D]^{-1} u_{n}
$$


With $Q$, the pressure field is calculated from

$$
p(\boldsymbol{r})=j \omega \rho \sum_{i=1}^{N} g\left(\boldsymbol{r}, \boldsymbol{r}_{w_{i}}\right) Q_{i} \quad \boldsymbol{r} \in \Gamma \cup \Omega_{f} \quad \boldsymbol{r}_{w_{i}} \in \Gamma^{\prime},
$$

thanks to the linearized Euler equation. The WSM exhibits non-uniqueness at critical wave numbers (Wilton et al., 1993) connected to the virtual shape defined by the superposition sources (Chen, 2006, Leblanc et al., 2010).

While there is much scope for discussion on the number and location of the interior sources for optimum accuracy, some authors as Alves (Alves, 2003) have established some useful rules of thumb, which are used in this paper.

\subsection{Irregular frequencies}

At frequencies corresponding to a natural frequency of the inner surface, HIE and WSM possess indeterminate solutions. Theoretically, the determinant of the final linear system is zero. Because of the body surface discretization (HIE) or the location of the superposition sources (WSM), the determinant is not exactly equal to zero and leads to a false solution. Moreover, the computational approximations generate an interval around the irregular frequency where the solution is not correct. Since the number of irregular frequencies increases rapidly with frequency (Courant et al., 1953), the error intervals can overlap. So, it is generally necessary - for medium or high frequency problems - to eliminate the indeterminations.

To highlight this unwanted behaviour, three different geometries immersed in water are studied ( $c=1500 \mathrm{~m} / \mathrm{s}$ ): (i) an infinite cylinder, (ii) a finite right circular cylinder and (iii) a sphere. In the first case, irregular wavenumbers associated to the interior Dirichlet problem are solutions of (Wilton et al., 1993)

$$
J_{n}\left(k a_{s}\right)=0
$$

where $J_{n}$ is the $n^{\text {th }}$ Bessel function and $a_{s}$ is the radius of the circle defined by superposition sources. In the second case, the critical frequencies are (Schenck, 1968)

$$
k_{m n q}=\sqrt{\left(\frac{m \pi}{2 b}\right)^{2}+\left(\frac{\alpha_{n q}}{a}\right)^{2}},
$$

(see Figure 2) in which $m$ is a positive integer and $\alpha_{n q}$ is the $q^{\text {th }}$ root of $J_{n}$ (Abramowitz et al., 1964). In the third case, irregular frequencies are given by (Schenck, 1968)

$$
k_{n q}=\frac{\alpha_{n q}}{a}
$$

where $a$ is the radius of the sphere. Here $\alpha_{n q}$ is the $q^{\text {th }}$ root of the $n^{\text {th }}$ spherical Bessel function $j_{n}$ (Abramowitz et al., 1964). 


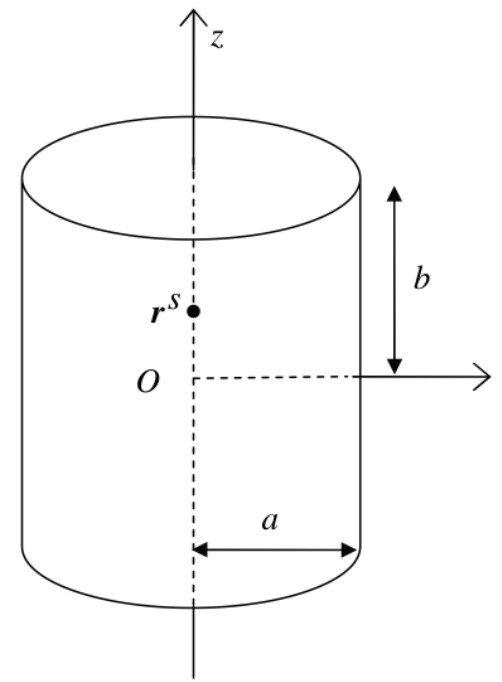

Figure 2. Finite right circular cylinder

\subsection{Unique solution by an average process}

The numerical technique proposed is very easy to implement. The idea is to perform two computations: one at the complex wavenumber $k-i \varepsilon$ and one other at $k+i \varepsilon$ where $\varepsilon$ is a small real number compared to $k$. Both two solutions are unique and are not affected by irregular frequencies effects. Indeed, these two frequencies are complex and can not coincide with eigenvalues of the interior problem that are real. To recover the original value of the frequency, the final pressure is obtained following average:

$$
p(\boldsymbol{r}, k)=\frac{p(\boldsymbol{r}, k-i \varepsilon)+p(\boldsymbol{r}, k+i \varepsilon)}{2} .
$$

The main difficulty is to evaluate the magnitude of $\varepsilon$ : both the shift must be small enough to assure the accuracy of the interpolation and high enough to remove the effect of the irregular frequency. To this end, we have developed a numerical tool in which HIE and WSM have been implemented using MatLab ${ }^{\mathrm{TM}}$ programming. When the variables exhibit an implicit $e^{-i \omega t}$ dependence, the first calculated frequency matches with an amplified oscillation and the second one with a damped oscillation. 


\section{Numerical investigations}

\subsection{Point-source check}

The numerical validation is realized using the point-source check (Schenck, 1968). This test consists in locating one point $\boldsymbol{r}^{s}$ into the geometry (e.g. see Figure 2). The source radiates a spherical analytical pressure with amplitude $M$ :

$$
p_{a}(\boldsymbol{r})= \pm M \frac{e^{i k\left|\boldsymbol{r}-\boldsymbol{r}^{s}\right|}}{\left|\boldsymbol{r}-\boldsymbol{r}^{s}\right|}= \pm M \frac{e^{i k D}}{D}
$$

from which its normal derivative value is evaluated on the surface:

$$
\frac{\partial p_{a}(\boldsymbol{r})}{\partial \boldsymbol{n}}= \pm M \frac{e^{i k D}}{D^{2}}\left(i k-\frac{1}{D}\right)\left(\boldsymbol{r}^{s}-\boldsymbol{r}\right) \cdot \boldsymbol{n}
$$

The nodal surface value vector $\partial p_{a} / \partial \boldsymbol{n}$ is introduced in [7]. Then, the system is solved and calculated pressure is compared to the analytical pressure by the meansquare error (MSE):

$$
\mathrm{MSE}=\sqrt{\frac{\sum_{n=1}^{N}\left|p_{c}\left(\boldsymbol{r}_{n}\right)-p_{a}\left(\boldsymbol{r}_{n}\right)\right|}{\sum_{n=1}^{N}\left|p_{a}\left(\boldsymbol{r}_{n}\right)\right|}}
$$

where $N$ is the total number of nodes of the mesh, $p_{c}\left(\boldsymbol{r}_{n}\right)$ and $p_{a}\left(\boldsymbol{r}_{n}\right)$ are respectively the calculated and the analytical pressure at the node $n$. Two or more point-sources in the same geometry is possible because analytical pressures are cumulative.

\subsection{Computations}

\subsection{1. $2 D$ case using the wave superposition method}

Working on an infinite cylinder (cf. Figure 3) with the WSM highlights the benefit obtained by the averaging process. Using the same shape but retracted (radius $a_{s}=a / 10$ ) for the superposition sources, the point-source check (located at $x=0.05 a \quad y=0.1 a$ ) is performed by averaging WSM results with the complex wave numbers as defined in Equation [15] for $\varepsilon$ ranging from $10^{-5}$ to $10^{-1}$. The number of superposition sources is defined by the distance between two adjoining superposition sources which is set as a quarter of the wavelength. Figure 4 shows the domain of validity of the averaging process for the $2^{\text {nd }}$ irregular wavenumber $k a_{s}=3.832$. The two following Figures 5 and 6 illustrate the influence of the coefficient $\varepsilon$. If $\varepsilon$ is chosen too high, the damping effect on resonance will be to important, leading to a constant but high errors. At the other end, selecting a small $\varepsilon$ will affect only the upper peaks of the spectra. Figure 5 can be viewed as an acceptable case of sufficient 
damping while for Figure 6 is clearly a lower limit for $\varepsilon$ : the overall error due to irregular wavenumber is unaltered except at those frequencies where the result is as good if better than for a greater $\varepsilon$ (cf. Figure 4).

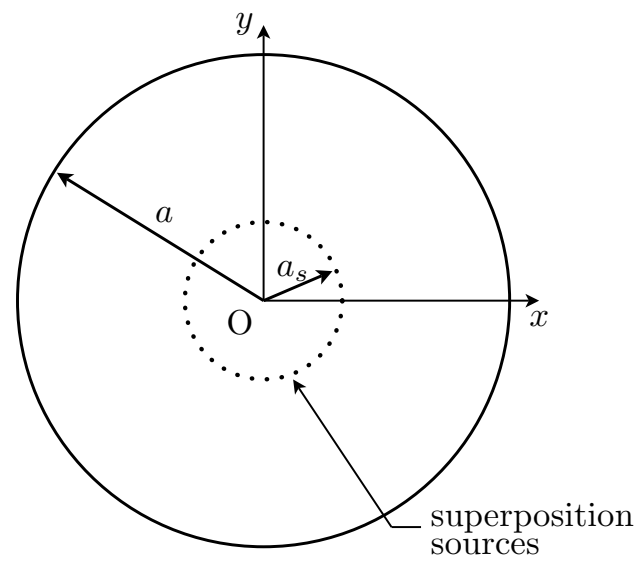

Figure 3. 2D representation of an infinite cylinder (radius a), with superposition sources located on a circle of radius $a_{s}$

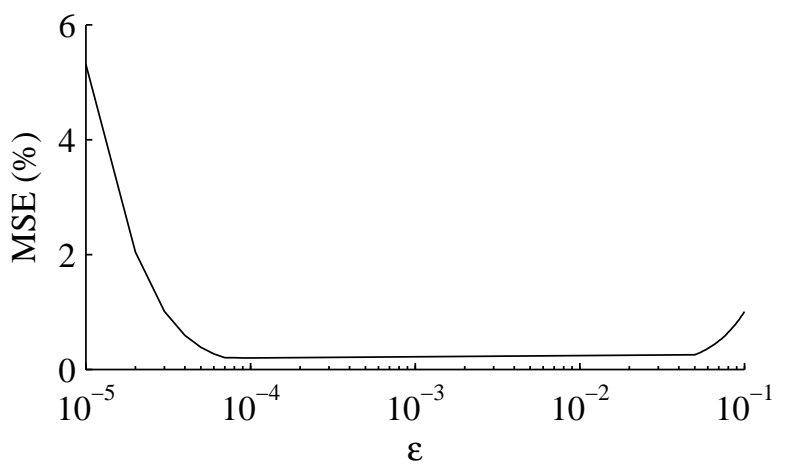

Figure 4. Point-source check: MSE obtained for the infinite cylinder and with averaged WSM: $10^{-5}<\varepsilon<10^{-1}$ and $k a_{s}=3.832$ 


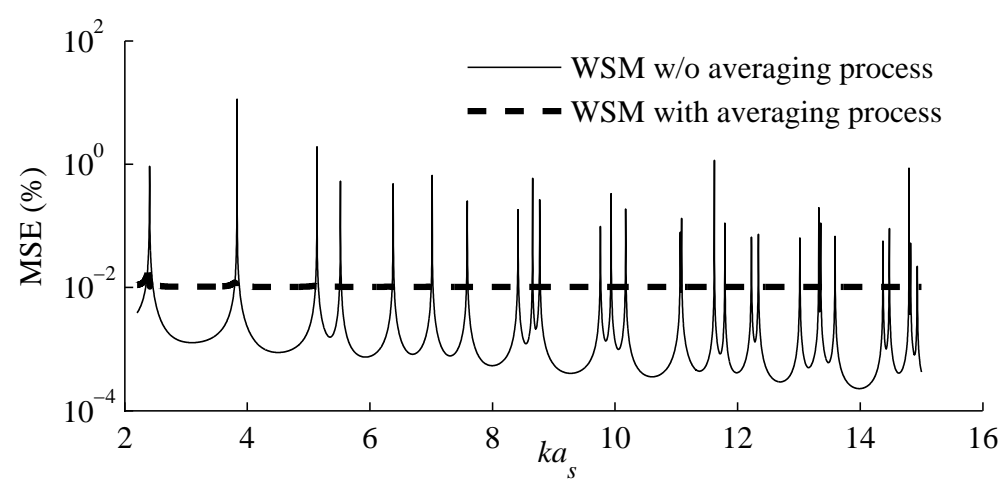

Figure 5. Point-source check: MSE obtained for the infinite cylinder and with averaged WSM: $\varepsilon=10^{-2}$ and $2<k b<15$

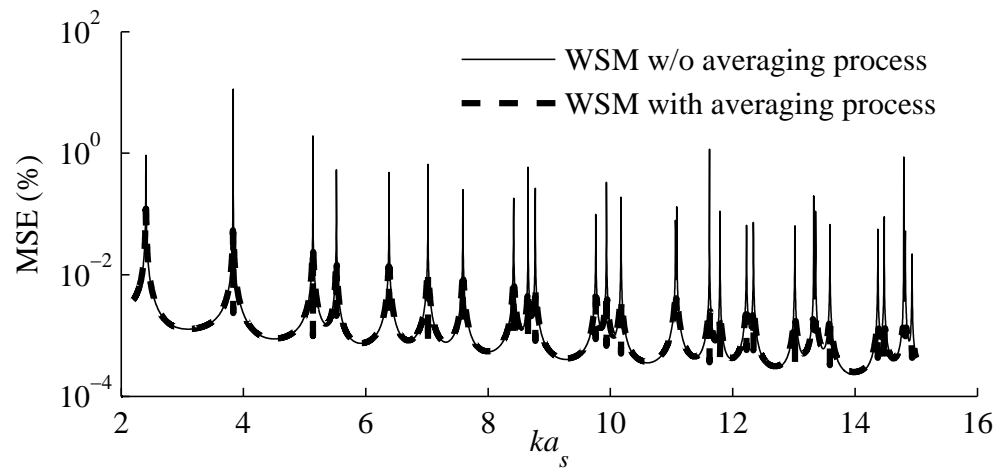

Figure 6. Point-source check: MSE obtained for the infinite cylinder and with averaged WSM: $\varepsilon=10^{-3}$ and $2<k b<15$

\subsection{2. $3 D$ case using the Helmholtz integral equation}

The first studied geometry with HIE is the finite right circular cylinder with $b=1.5 a$. This choice has the effect of shifting the irregular frequencies in the lowfrequency range. We have selected three critical wavenumbers such that

$$
\begin{aligned}
& k_{1} a=k_{101} a=\sqrt{\left(\frac{\pi}{3}\right)^{2}+\alpha_{01}^{2}} \\
& k_{2} a=k_{102} a=\sqrt{\left(\frac{\pi}{3}\right)^{2}+\alpha_{02}^{2}} \\
& k_{3} a=k_{703} a=\sqrt{\left(\frac{7 \pi}{3}\right)^{2}+\alpha_{03}^{2}} .
\end{aligned}
$$

$k_{1}, k_{2}$ and $k_{3}\left(k_{3} a \simeq 11.341\right)$ are the $1^{\text {st }}$, the $10^{\text {th }}$ and the $100^{\text {th }}$ values given by Equation [13] and are symmetric modes with respect to the symmetry plane. We have 
chosen a point-source test that is consistent with the symmetries of the three above selected frequencies (axisymmetry and plane symmetry):

- two sources on the axis of the cylinder: $\boldsymbol{r}^{s_{1}}$ (resp. $\boldsymbol{r}^{s_{2}}$ ) such as $z=b / 3$ (resp. $z=-b / 3)$ and amplitude $M($ resp. $M)$.

The mesh is built in order to respect the " $\lambda / 4$ criterion", which states that the dimension of the largest element of the mesh has to be minor than a quarter of the wavelength to get accurate solutions (Zienkiewicz, 1971). The mesh is limited to the meridian of the cylinder. It is made of identical 3-nodes elements: 8 elements on the radius $a$ and 12 elements on the half height $b$.

The second geometry is the sphere. The chosen reduced wavenumber is such that $k_{19}{ }_{1} a=\alpha_{191} \simeq 24.878$. The point-source test is consistent with the axisymmetry and plane symmetry of the associated mode:

- two sources on the axis of the sphere: : $\boldsymbol{r}^{s_{1}}$ (resp. $\boldsymbol{r}^{s_{2}}$ ) such as $z=a / 4$ (resp. $z=-a / 4)$ and amplitude $M(\operatorname{resp} . M)$.

The mesh is sized to respect the " $\lambda / 4$ criterion": 503 -nodes elements on the meridian. Because of the quadratic variation of the elements, the meridian is approximated by set of connected parabolic curves that very slightly differs from the half circle. So, critical frequencies are very slightly shifted up. A preliminary numerical study has allowed to locate with accuracy the value of the numerical critical wavenumber associated with $k_{191}$ :

$$
k a=25.013 \quad \text { where } \quad \mathrm{MSE}=774 \% .
$$

Others choices of frequencies are obviously possible and don't alter the course of the study below. The average approximation is evaluated by varying the parameter $\varepsilon$ between $k / 10$ and $k / 1000$.

A comprehensive study is achieved for the cylinder. The MSE obtained from the HIE with the above test is:

- for $k_{1}: \mathrm{MSE}=1764 \%$;

- for $k_{2}: \mathrm{MSE}=6301 \%$;

- for $k_{3}: \mathrm{MSE}=592 \%$.

This illustrates the effect of these irregular frequencies. Results are shown in Tables 1,2 and 3 for $k_{1}, k_{2}$ and $k_{3}$.

Table 1. MSE of the average process depending of the parameter $\varepsilon$. Finite right circular cylinder and $k_{1}$

\begin{tabular}{|c|c|c|c|c|c|c|c|}
\hline$\varepsilon$ & $k_{1} / 10$ & $k_{1} / 20$ & $k_{1} / 50$ & $k_{1} / 100$ & $k_{1} / 200$ & $k_{1} / 500$ & $k_{1} / 1000$ \\
\hline average (\%) & 5.98 & 1.47 & 0.25 & 0.14 & 0.12 & 0.18 & 36.45 \\
\hline
\end{tabular}


Table 2. MSE of the average process depending of the parameter $\varepsilon$. Finite right circular cylinder and $k_{2}$

\begin{tabular}{|c|c|c|c|c|c|c|c|}
\hline$\varepsilon$ & $k_{2} / 10$ & $k_{2} / 20$ & $k_{2} / 50$ & $k_{2} / 100$ & $k_{2} / 200$ & $k_{2} / 500$ & $k_{2} / 1000$ \\
\hline average (\%) & 34.68 & 8.22 & 1.61 & 0.96 & 0.90 & 0.89 & 0.89 \\
\hline
\end{tabular}

Table 3. MSE of the average process depending of the parameter $\varepsilon$. Finite right circular cylinder and $k_{3}$

\begin{tabular}{|c|c|c|c|c|c|c|c|}
\hline$\varepsilon$ & $k_{3} / 10$ & $k_{3} / 20$ & $k_{3} / 50$ & $k_{3} / 100$ & $k_{3} / 200$ & $k_{3} / 500$ & $k_{3} / 1000$ \\
\hline average (\%) & 163.65 & 31.85 & 4.84 & 1.38 & 0.70 & 0.70 & 1.38 \\
\hline
\end{tabular}

We observe that the average process is very efficient when $\varepsilon$ is around $k / 200$. Pressure versus nodes number is given in Figures 7, 8 and 9. Normalized analytical surface pressure is compared to the normalized pressure $p / M$ with $\varepsilon=k / 200$. The abscissa axis designates the numbering of the equidistant nodes of the half meridian: node 1 is at the centre of an endcap of the cylinder and node 41 is on the symmetry plane. In these figures, analytical and average pressures are superimposed. This proves the very good accuracy of the average technique.
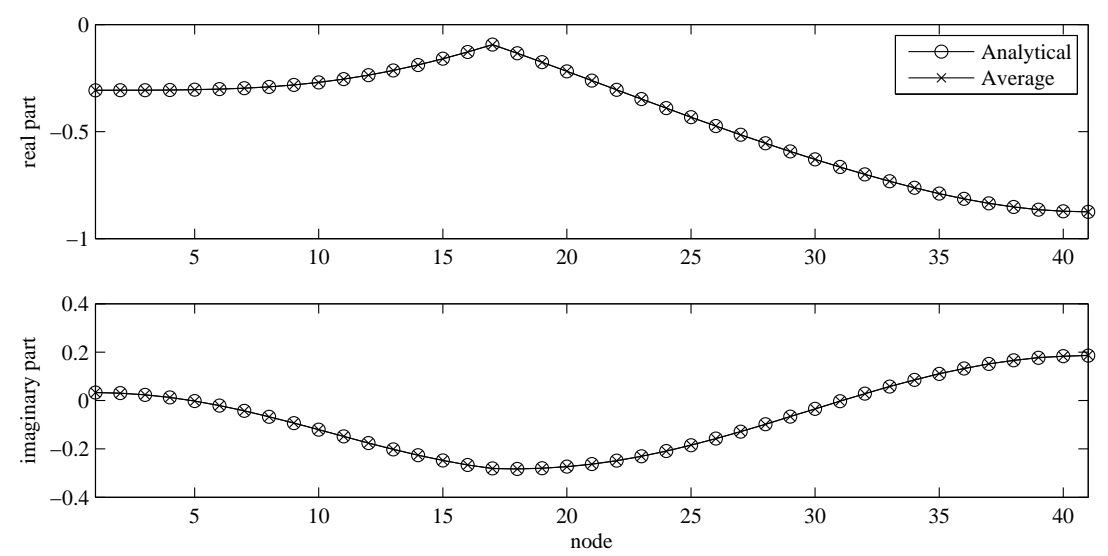

Figure 7. Comparison of normalized surface pressures on the half meridian of the finite right circular cylinder at $k_{1}$

The sphere is directly tested with the average process and $\varepsilon=k / 200$ at $k$ given by Equation [20]. Results are summarized in MSE $=0.76 \%$. Normalized analytical surface pressure is compared to the normalized average pressure on Figure 10. An 

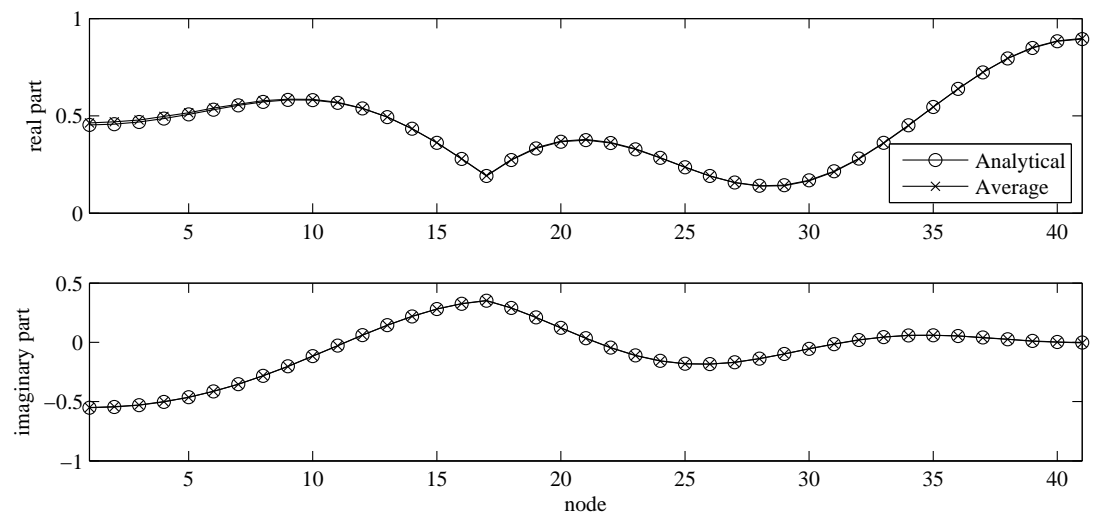

Figure 8. Comparison of normalized surface pressures on the half meridian of the finite right circular cylinder at $k_{2}$
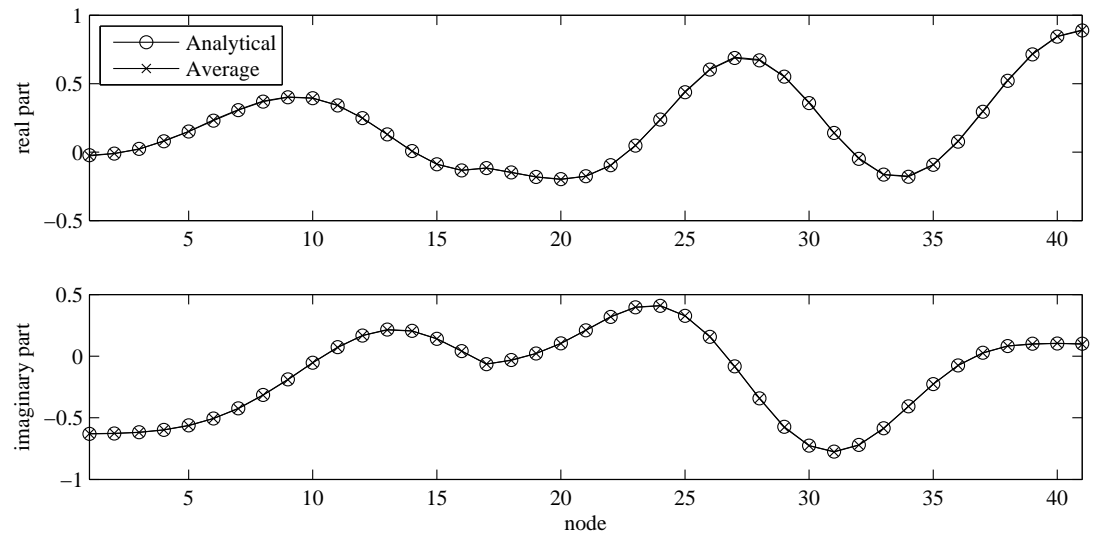

Figure 9. Comparison of normalized surface pressures on the half meridian of the finite right circular cylinder at $k_{3}$

excellent concordance is observed between analytical values and results issued from the average method. Moreover, these results confirm the choice of $\varepsilon$. 

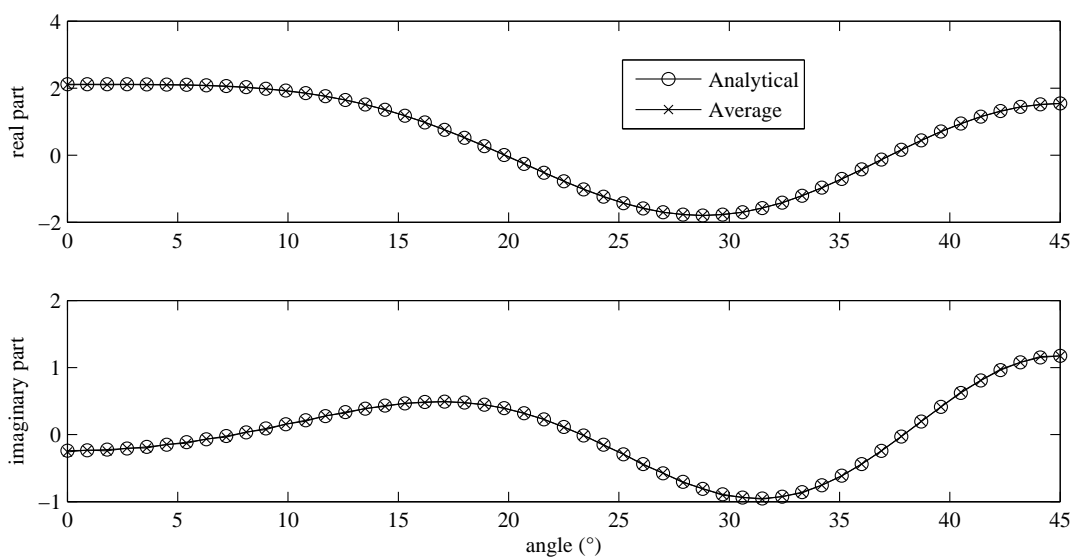

Figure 10. Analytical normalized surface pressure compared to average pressure on the half meridian of the sphere at $k a=25.013$

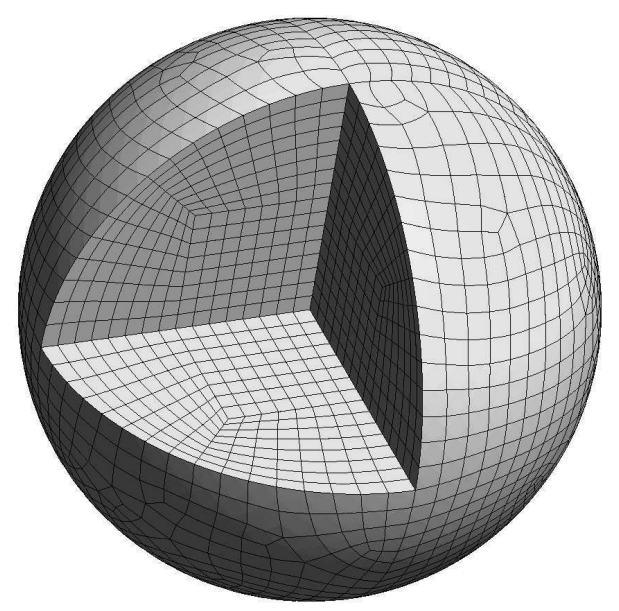

Figure 11. Mesh of the cat's eye model

\section{Cat's eye application}

More and more authors consider the cat's eye geometry as a reference (Marburg et al., 2008, Marburg et al., 2005, Makarov et al., 1998, Schneider et al., 2003). It consists of a sphere with radius $R$ where the positive octant (i.e. where the cartesian coordinates are such that $x \geq 0, y \geq 0, z \geq 0$ ) is cut out (see Figure 11). The radiation problem in air $(c=340 \mathrm{~m} / \mathrm{s})$ is investigated with HIE for a vibrating surface 
where it coincides with the spherical one. The plain surfaces of the missing octant remain calm. The idea is that the sound pressure at the centre of the backside should behave asymptotically (as frequency increases) like one at the surface of the sphere. This backside point is the opposite point of the missing octant and is defined in spherical coordinates such as $(r, \vartheta, \varphi)=\left(R, 135^{\circ}, 225^{\circ}\right)$. Another particular point under consideration is the centre point $R=0$. Besides, this radiator allows construction of a smooth solution that will make it easy to identify solution failures caused by the ill-conditioning of the integral operator associated to the irregular frequencies. Due to the complexity of the geometry, we expect more irregular frequencies than a sphere. The normal particle velocity for the vibrating surface is uniform. In this case, the sound pressure at the backside point tends towards the pressure of a pulsating sphere:

$$
p(R)=\rho c v_{n} \frac{k R}{\sqrt{1+k^{2} R^{2}}} .
$$

Simultaneously, a point-source check is driven with two sources: $\boldsymbol{r}^{s_{1}}$ (resp. $\boldsymbol{r}^{s_{2}}$ ) is defined by $(r, \vartheta, \varphi)=\left(R / 2,135^{\circ}, 210^{\circ}\right)\left(\operatorname{resp} .(r, \vartheta, \varphi)=\left(R / 2,135^{\circ}, 240^{\circ}\right)\right)$ and amplitude $M$ (resp. $2 M$ ). The mesh satisfies the " $\lambda / 4$ criterion" up to about $k R=18$ and the computation are carried out up to $k R=27.720$. It is made of 2324 isoparametric quadratic 8-nodes elements. The whole frequency range is covered by 3001 equidistant computation frequencies. The average process defined by Equation [15] is used with $\varepsilon=k / 200$.

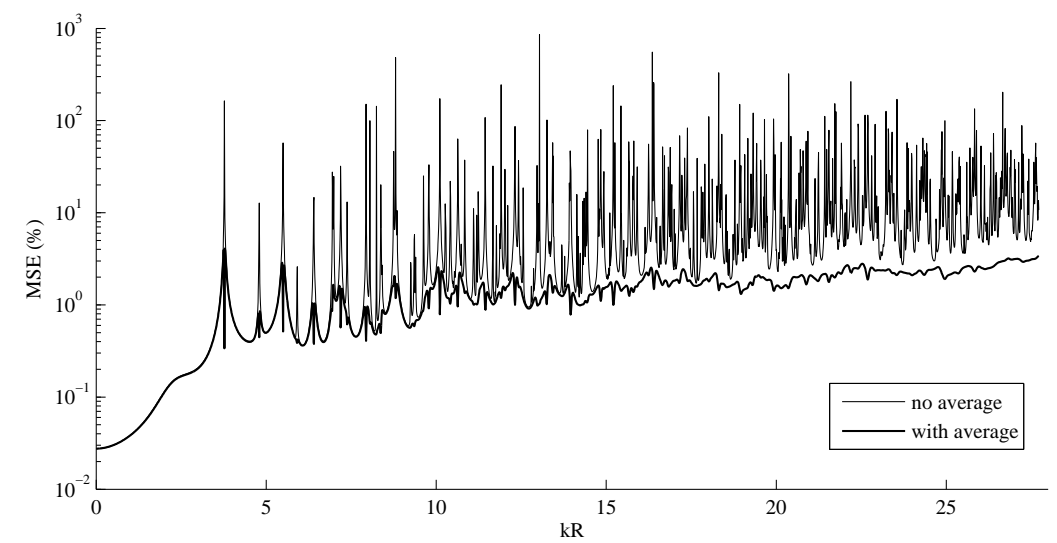

Figure 12. MSE of the cat's eye

Results are given in Figures 12, 13 and 14 (some peaks are truncated because of their large amplitude). The failure at the irregular frequencies are corrected by the average process. The MSE slowly increases with the frequency because the mesh is the same throughout the frequency range. As expected, the behaviour of the normalized backside pressure tends toward 1 with small periodic oscillations in Figure 13. In 

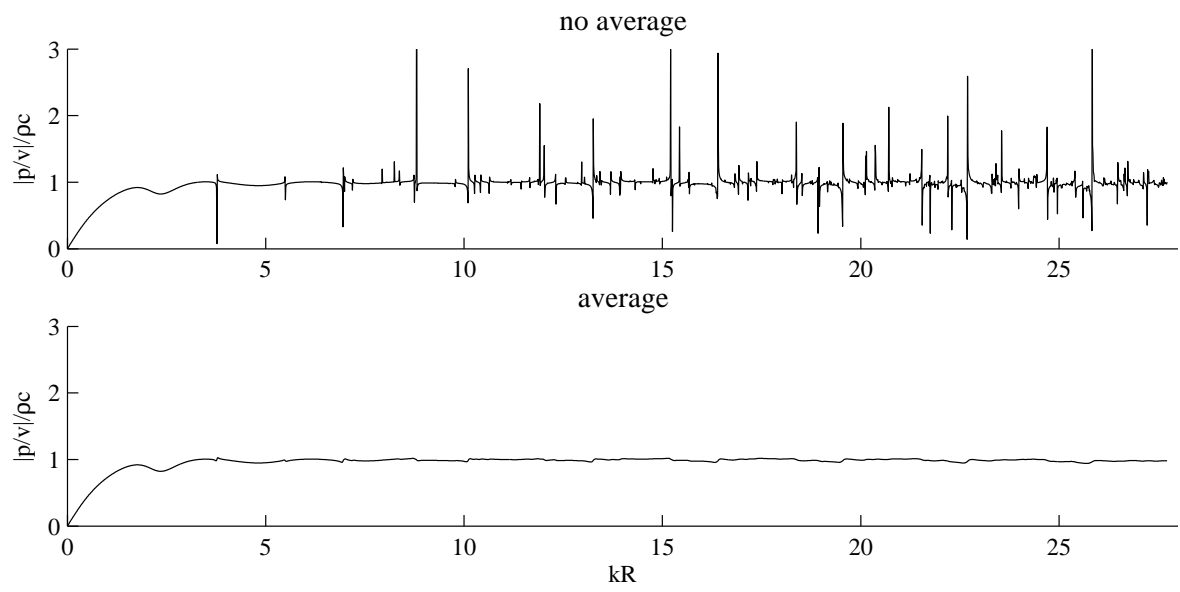

Figure 13. Pressure at the backside point of the cat's eye
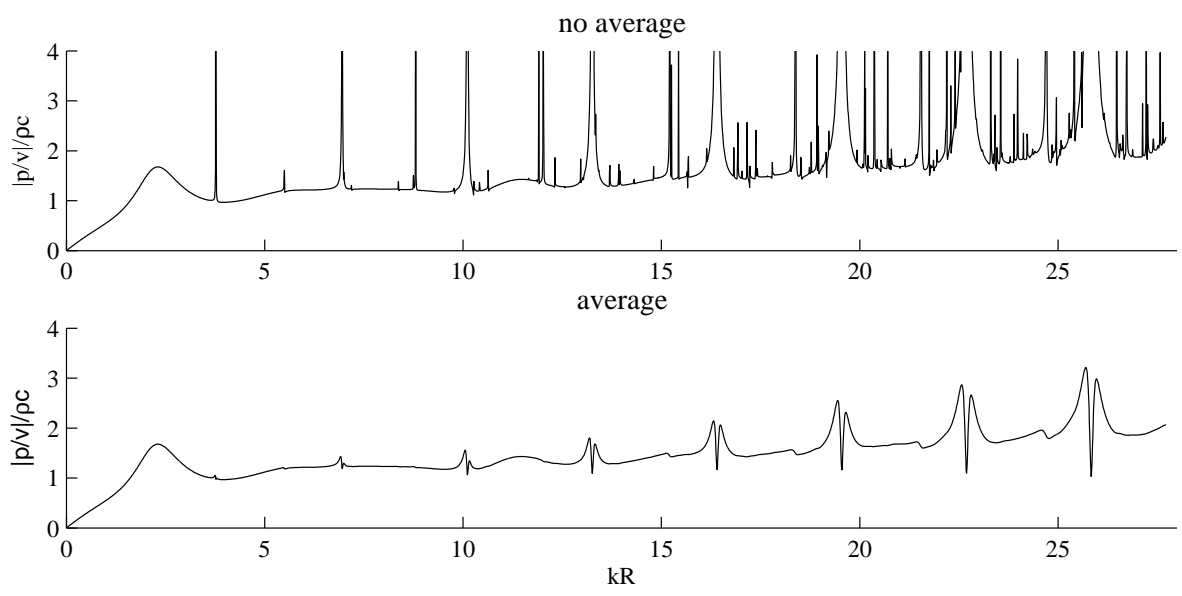

Figure 14. Pressure at the centre point of the cat's eye

Figure 14, these periodic oscillations are resumed with amplitude getting higher with frequency and are probably due to resonances. Finally, $\varepsilon=k / 200$ provides good results over the entire frequency range. 


\section{Conclusion}

HIE and WSM do not provide correct solution for exterior acoustic Neumann problem when the wavenumber is equal or in the neighborhood of an eigenvalue of the associated interior Dirichlet problem. The proposed solution is based upon an average of pressures between the computational frequency minored and majored by an imaginary factor $i \varepsilon$ (cf. Equation [15]). The average process has been successfully applied to the WSM in the case of the infinite cylinder while too more computations concern the HIE. For a finite right circular cylinder, computations have shown the ability of the average process to remove the irregular frequencies effects. The study of the sphere confirm its validity and its efficiency. Moreover, this procedure was applied to the HIE to calculate the acoustic radiation of the complex 3D cat's eye.

Up to now, there is no theoretical justification so preliminary study is necessary in order to determine $\varepsilon$. From numerical validations, this technique is efficient and very easy to implement on a large frequency range and for complex geometries.

In this paper, development and calculation concern only radiation problems. Nevertheless, extension to scattering problem is straightforward by adding incident pressure in the second member of Equation [4].

\section{References}

Abramowitz M., Stegun I. A., Handbook of Mathematical Functions with Formulas, Graphs, and Mathematical Tables, Dover Publications, New York, 1964.

Alves C. J. S., « The method of fundamental solutions for scattering and radiation problems », Engng Anal Bound Elem, vol. 27, p. 759-769, 2003.

Benthien W., Schenck A., « Nonexistence and nonuniqueness problems associated with integral equation methods in acoustics », Comput. Struct., vol. 65, n 3, p. 295-305, 1997.

Brebbia C. A., Boundary Element Methods in Acoustics, Springer, 1991.

Burton A. J., Miller G. F., « The application of integral equation methods to the numerical solution of some exterior boundary-value problems », Proc. Roy. Soc. London, vol. A323, p. 201-210, 1971.

Chen I. L., « Using the method of fundamental solutions in conjunction with the degenerate kernel in cylindrical acoustic problems », J. Chin. Inst. Eng., vol. 29, n 3, p. 445-457, 2006.

Chen Z. S., Hofstetter G., Mang H. A., « A symmetric Galerkin formulation of the boundary element method for acoustic radiation and scattering », J. Comput. Acoust., vol. 5, p. 219241, 1997.

Copley L. G., « Fundamental Results Concerning Integral Representations in Acoustic Radiation », J. Acoust. Soc. Am., vol. 44, p. 41-58, 1968.

Courant R., Hilbert D., Methods of Mathematical Physics, vol. 1, Wiley, New York, 1953.

Fairweather G., Karageorghis A., Martin P. A., « The method of fundamental solutions for scattering and radiation problems », Eng. Anal. Boundary Elem., vol. 27, p. 759-769, 2003. 
Jones D. S., «Integral equations for the exterior acoustic problem », Q. J. Mech appl. Math, vol. XXVII, p. 129-142, 1974.

Juhl P., «A numerical study of the coefficient matrix of the boundary element method near characteristic frequencies », J. Sound Vib., vol. 175, p. 39-50, 1994.

Koopmann G. H., Song L., Fahnline J. B., « A method for computing acoustic fields based on the principle of wave superposition », J. Acoust. Soc. Am., vol. 86, n 6, p. 2433-2438, December, 1989.

Lavie A., Modélisation du rayonnement ou de la diffraction acoustique par une méthode mixte équations intégrales-champ nul, $\mathrm{PhD}$ thesis, Université des Sciences et Techniques de Lille, 1989.

Leblanc A., Ing R. K., Lavie A., « A Wave Superposition Method Based on Monopole Sources with Unique Solution for All Wave Numbers », Acta Acustica united with Acustica, vol. 96, p. 125-130, 2010.

Makarov S. N., Ochmann M., « An iterative solver of the Helmholtz integral equation for highfrequency acoustic scattering », J. Acoust. Soc. Am., vol. 103, p. 742-750, February, 1998.

Marburg S., Amini S., «Cat's eye radiation with boundary elements: comparative study on treatment of irregular frequencies », J. Comput. Acoust., vol. 13, n 1, p. 21-45, 2005.

Marburg S., Nolte B., Acoustics of Noise Propagation in Fluids: Finite and Boundary Element Methods, Springer, 2008.

Marburg S., Schneider S., "Performance of iterative solvers for acoustic problems. Part I. Solvers and effect of diagonal preconditioning », Eng. Anal. Boundary Elem., vol. 27, n 7, p. 727 - 750, 2003. Special issue on Acoustics.

Marschall R. A., « Boundary element solution of a body's exterior acoustic field near its internal eigenvalues », J. Comput. Acoust., vol. 1, p. 335-353, 1993.

Meyer W. L., Bell W. A., Zinn B. T., Stalybrass M. P., « Boundary integral solutions of three dimensional acoustic radiation problems », J. Sound Vib., vol. 59, p. 245-262, July, 1978.

Schenck H. A., «Improved integral formulation for acoustic radiation problems », J. Acoust. Soc. Am., vol. 44, p. 41-58, 1968.

Schneider S., Marburg S., « Performance of iterative solvers for acoustic problems. Part II. Acceleration by ILU-type preconditioner », Eng. Anal. Boundary Elem., vol. 27, n 7, p. 751 - 757, 2003. Special issue on Acoustics.

Segalman D. J., Lobitz D. W., SuperCHIEF: a modified CHIEF method, Technical Report $n$ SAND-90-1266, SANDIA Labs, 1990.

Segalman D. J., Lobitz D. W., «A method to overcome computational difficulties in the exterior acoustics problem », J. Acoust. Soc. Am., vol. 91, n 4, p. 1855-1861, 1992.

Seybert A. F., Rengarajan T. K., « The use of CHIEF to obtain unique solutions for acoustic radiation using boundary-integral equations », J. Acoust. Soc. Am., vol. 81, n S1, p. S99S99, 1987.

Seybert A. F., Soenarko B., Rizzo F. J., Shippy D. J., « An advanced computational method for radiation and scattering of acoustic waves in three dimensions », J. Acoust. Soc. Am., vol. 77, p. 362-368, 1985.

Stupfel B., Lavie A., Decarpigny J.-N., « Combined integral equation and null-field method for the exterior acoustic problem », J. Acoust. Soc. Am., vol. 83, p. 927-941, 1988. 
Waterman P. C., « New Formulation of Acoustic Scattering », J. Acoust. Soc. Am, vol. 45, n 6, p. 1417-1429, 1969.

Wilcox C. H., «A generalization of theorems of Rellich and Atkinson », Proc. Am. Math. Soc., vol. 7, p. 271-276, 1956.

Wilton D. T., Mathews I. C., Jeans R. A., « A clarification of nonexistence problems with the superposition method », J. Acoust. Soc. Am, vol. 94, n 3, p. 1676-1680, September, 1993.

Wu T. W., Seybert A. F., « A weighted residual formulation for the CHIEF method in acoustics », J. Acoust. Soc. Am., vol. 90, n 3, p. 1608-1614, 1991.

Yang S. A., « An integral equation approach to three-dimensional acoustic radiation and scattering problems », J. Acoust. Soc. Am., vol. 116, p. 1372-1380, September, 2004.

Zienkiewicz O. C., The finite element method in engineering science, McGraw-Hill, Maidenhead, 1971. 